\title{
Measuring outpatient resource use and case mix in ophthalmology in north east England
}

\author{
Paul McNamee, David Parkin, David Allen, Nick Steen, Allen Hutchinson
}

Centre for Health Services Research, School of Health Sciences, University of Newcastle, Newcastle upon Tyne $\mathrm{P}$ McNamee

N Steen

Department of Epidemiology and Public Health, Schoo of Health Sciences, University of Newcastle, Newcastle upon Tyne

D Parkin

Sunderland Eye Infirmary, Queen Alexandra Road, Sunderland

D Allen

School of Health and Related Research, University of Sheffield, Sheffield

A Hutchinson

Correspondence to: Dr D Parkin, Department of Epidemiology and Public Health, The Medical School, School of Health Sciences, University of Newcastle, Newcastle upon Tyne NE2 $4 \mathrm{HH}$, United Kingdom.

Accepted for publication 5 June 1997

\begin{abstract}
Objectives-To test the extent to which two existing ambulatory case mix measures (Ambulatory Visit Groups and Ambulatory Patient Groups) and other variables can explain resource use variations in ophthalmic outpatient visits.

Design-Three week prospective study of three consultant outpatient clinics.

Setting-One ophthalmic hospital (Sunderland Eye Infirmary, Sunderland, Tyne and Wear) and three outreach clinics (South Tyneside District Hospital, South Shields, Tyne and Wear; Dryburn Hospital, Durham, Co Durham; and Hartlepool General Hospital, Hartlepool, Cleveland). Subjects-325 patients who visited ophthalmic outpatient clinics.

Main outcome measures-Mean consultation time and mean cost distributions by case mix group, analysed by analysis of variance.

Results-Ambulatory case mix measures can explain some of the variation in resource use for outpatient visits, but different measures differ in the extent to which they can do so. Clinicians' behaviour also accounts for a significant amount of such variation. Simpler measures of visit type, without diagnostic or procedure information, do not explain resource use variations.

Conclusions-Existing measures perform reasonably well, but their data requirements may preclude their introduction in the National Health Service. Caution is required in advocating simpler measures, however. The influence of clinical practice on resource use variations is important; in this study, most differences between clinicians were not attributable to differences in case mix.
\end{abstract}

\section{(F Epidemiol Community Health 1998;52:247-252)}

Knowledge of the range of activities and the costs of health care services is essential for promoting efficient health care delivery. Such information can be produced in many ways, but one useful method is to condense the enormous variety of hospital patient cases into clinically similar groups that use similar amounts of resources. Substantial international experience in this field of case mix measurement has been gained using Diagnosis Related Groups (DRGs), ${ }^{1}$ and the process of measurement has been facilitated in the National Health Service (NHS) for inpatient care by the creation of Healthcare Resource Groups
(HRGs). ${ }^{2}$ Development of measures for outpatient care has hitherto not been a priority, primarily because the acute sector involves much higher costs, but also because there are many conceptual and practical difficulties involved in capturing and classifying clinical activity data in outpatients. ${ }^{3}$ However, recent developments suggest that extension of case mix measurement to the outpatient setting may now be desirable. ${ }^{4}$

Firstly, there is pressure on hospitals to manage more patients in ambulatory (day care, day case, and outpatient) care. Better patient based information on activities and costs is therefore required, for the planning, monitoring, and resourcing of such care. Secondly, the system of contracts between providers and health care commissioning organisations and general practitioner fund holders means that there is now a need to define, identify, and classify the nature of outpatient services.

Continuing refinement of outpatient contracts only serves to heighten the requirement to have case mix information available. There may be advantages to both providers and purchasers in the ability to establish different prices for outpatient visits according to the character of the visit; for example, whether a procedure was undertaken, whether the visit involved a diagnostic assessment, or whether a visit was simply to monitor patient management.

Researchers in the United States have developed several ambulatory case mix measures, notably Ambulatory Visit Groups (AVGs) ${ }^{5}$ and Ambulatory Patient Groups (APGs). ${ }^{6}$ The purposes and characteristics of these systems have been described elsewhere.$^{7-9}$ Briefly, AVGs were developed using clusters of related diagnoses, with groups subdivided on the basis of variations in costs for procedure groups, or variations in physician time for medical groups. APGs were derived on the basis of cost variation, after clustering of clinically related ambulatory procedures (procedure APGs), and clinically similar ICD-9-CM diagnoses (medical APGs). Thus, the objective of both systems is to explain variations in resource use rather than identify what resource use ought to be.

However, an essential difference between them is that AVGs were designed as product definitions of office based specialists' services, while APGs were created for prospective reimbursement for hospital based outpatient care. As a result, APGs cover a wider range of procedures and, unlike AVGs or DRGs, permit cases to be assigned more than one procedure group. Although AVGs have not been used in practice, 
several APG projects have been undertaken, for example in Australia. ${ }^{10}$

These ambulatory measures could be adapted to create a system for the NHS, in a similar way to that by which DRGs were used as a basis for developing HRGs. ${ }^{11}{ }^{12}$ They rely on diagnostic and procedure data, however, which are not routinely collected in NHS outpatient clinics. Thus, consideration of these measures would need to bear in mind the question of information availability.

The problem of data requirements does not by itself rule out use of either system. Both the costs and the benefits of implementing and using a case mix measure must be taken into account. An important prior question is the extent to which either measure is useful in a UK clinical practice setting. In particular, their ability to explain variations in resource use is perhaps the most important attribute to be assessed. As part of an evaluative study of ambulatory case mix measures conducted on behalf of the National Casemix Office, this paper describes how well each measure performed on an ophthalmology data set and discusses the usefulness of case mix for purchasing.

\section{Methods}

The study collected data in two stages from the clinics of three consultants at Sunderland Eye Infirmary, South Tyneside District Hospital, Dryburn Hospital, and Hartlepool General Hospital. Firstly, data were collected for one week in February 1993. An outpatient clinic data collection form was used, which was a modified version of a form already in routine use as an audit tool for new outpatient cases. The data collection method proved satisfactory and it was subsequently used in the main study, which was undertaken over two weeks in March and April 1993.

Data were collected on date of birth, sex, date seen, clinician seen, source of referral, visit type, diagnosis, investigations, procedures, clinician consultation time, nurse time, and management intention. Diagnoses were written in freehand text on the outpatient clinic form and coded to ICD-9 after the clinic sessions by an audit assistant. These were subsequently recoded to ICD-9-CM, as this is required by the AVG and $\mathrm{APG}$ grouping software.

The AVG and APG software also require procedures to be coded using a classification used for payment of ambulatory surgery in the United States, the Current Procedural Terminology, Version 4 (CPT- 4$).{ }^{13}$ This was also felt by the participating clinicians to describe outpatient clinical practice better than the OPCS-4 operative classification. Therefore, procedures were recorded using CPT- 4 descriptions and codes were added by the audit assistant after clinic sessions.

In addition, clinicians recorded the time they spent in face to face contact with patients, to the nearest minute. Nurse times were collected separately, using a system already in routine use in the hospital that records various activities that are allocated standard units of six minutes.

Cost data were produced by the Finance Department of Sunderland District General Hospital for a pre-specified list of procedures and investigations after data collection was complete. These were calculated by determining standard variable costs for quantities of consumables involved, using estimates obtained from supplies and pharmacy departments. Therefore, no allowances were made for indirect costs such as depreciation of machinery or clerical staff time.

In determining a cost for each patient visit, values for clinical and nurse time were added to procedure and investigation costs. Clinician costs for four grades of staff were based on the midpoint of average hourly cost from 1993/4 pay scales. These were subsequently translated into costs per minute, as follows:

\begin{tabular}{ll}
\hline $\begin{array}{l}\text { Consultant } \\
\text { (based on no distinction award) }\end{array}$ & 0.41 \\
$\quad$ Clinical assistant & 0.29 \\
$\begin{array}{l}\text { Senior registrar } \\
\text { (based on 32 ADHs class 3) }\end{array}$ & 0.19 \\
$\quad \begin{array}{l}\text { Senior house officer } \\
\text { (based on 32 ADHs class 3) }\end{array}$ & 0.15 \\
\hline
\end{tabular}

Nurse costs for five staff grades were based on the midpoint of average hourly cost from $1993 / 4$ pay scales. They were also translated into costs per minute.

All items of data were entered into a single database for analysis. From this, the relevant data, comprising diagnosis, procedures, age, and sex for grouping visits to AVGs and APGs, were extracted.

The ability of AVGs and APGs to explain variation in resource use was investigated using analysis of variance (ANOVA) with consultation time and visit cost as the dependent variables. Previous work ${ }^{14}$ indicated that considerable variability among clinicians could be expected. Therefore, differences between individual clinicians were taken account of in the model and the relative importance of case mix and individual clinician behaviour was assessed by fitting these two variables sequentially into the ANOVA model in both possible ordersclinician followed by case mix group and case mix group followed by clinician. To ensure sufficient number of cases within AVG and APG categories, groups containing less than eight cases were combined to form a single group.

Another aim was to assess whether the basic character of the outpatient visit, rather than its clinical characteristics, could be used in contract setting and pricing. Six patient type categories were constructed with input from the lead clinician before data collection, and tested in a similar fashion to AVGs and APGs. These categories were: new visit; review: diagnostic assessment; review: follow up after diagnostic assessment; review: procedure/ treatment; review: follow up after procedure; monitoring. Note that these categories do not require information on diagnosis or procedure. 
Table 1 Most frequent ambulatory visit groups (AVGs)

\begin{tabular}{|c|c|c|c|c|c|c|}
\hline \multirow[b]{2}{*}{$A V G$} & \multirow[b]{2}{*}{ Label } & \multirow[b]{2}{*}{$\%$ Visits } & \multicolumn{2}{|c|}{ Consultation time } & \multicolumn{2}{|l|}{ Cost } \\
\hline & & & Mean & $S D$ & Mean & $S D$ \\
\hline 0223 & Review, other anterior segment and optic nerve & 30.5 & 9.6 & 5.2 & 4.2 & 2.4 \\
\hline 0222 & Review, cataract,aphakia, pseudophakia & 19.4 & 10.6 & 6.0 & 4.3 & 3.0 \\
\hline 0202 & New, cataract, aphakia, pseudophakia & 6.5 & 13.9 & 5.4 & 5.8 & 2.5 \\
\hline 2302 & Screening, surveillance and symptoms & 6.2 & 11.5 & 6.5 & 7.3 & 8.6 \\
\hline 0224 & Review, retina, choroid and vitreous & 5.8 & 9.7 & 4.5 & 4.2 & 4.1 \\
\hline 0200 & New, external eye disorders & 5.2 & 11.0 & 9.1 & 4.6 & 3.7 \\
\hline 0220 & Review, external eye disorders & 4.3 & 5.7 & 2.9 & 2.8 & 1.6 \\
\hline 0225 & Review, visual disturbances, strabismus and motility disorders & 3.7 & 9.6 & 6.8 & 4.0 & 2.5 \\
\hline 0204 & New, retina, choroid and vitreous & 2.5 & 13.1 & 4.2 & 5.4 & 3.2 \\
\hline 0228 & Review, other diseases of the eye & 2.2 & 8.1 & 3.4 & 4.0 & 1.0 \\
\hline 0821 & Review, connective tissue disease & 1.8 & 7.0 & 4.7 & 3.5 & 2.8 \\
\hline 0205 & New, visual disturbances, strabismus and motility disorders & 1.8 & 14.2 & 4.7 & 7.9 & 4.3 \\
\hline 0245 & Procedure, anterior segment, other, class 1 & 1.5 & 19.6 & 4.2 & 7.9 & 3.6 \\
\hline 0264 & Procedure, diagnostic eye procedures, class 1 & 1.2 & 11.2 & 3.1 & 7.4 & 6.8 \\
\hline Others & & 7.4 & 12.3 & 7.7 & 10.0 & 13.0 \\
\hline All visits & & 100.0 & 10.6 & 6.2 & 5.3 & 5.5 \\
\hline
\end{tabular}

\section{Results}

Consultation times were recorded for 330 consecutive visits to the outpatient clinics of three consultants. Of these, five cases could not be assigned a valid AVG or APG or both because of missing diagnostic information. The analyses reported in the rest of this paper therefore cover 325 visits.

Thirty four different AVGs and 28 different APGs described total workload during the course of the study. Tables 1 and 2 show details of the most frequent AVGs and APGs. With AVGs, there was a more even spread of visits across different groups, with review patients making up the bulk of the workload. The most frequent AVGs were AVG 0223 (review, other anterior segment and optic nerve) and AVG 0222 (review, cataract, aphakia and pseudophakia), which together described around 50\% of all visits.

Mean consultation times varied from 5.7 minutes for AVG 0220 (review, external eye disorders) to 19.6 minutes for AVG 0245 (procedure, anterior segment, other, class 1). Average visit cost varied from $£ 2.79$ for AVG 0220 to $£ 10.01$ for the "other" AVGs, which contained high costs for two AVGs in particular: AVG 0255 (procedure, plastic and reconstructive eye, class 3) and AVG 0265 (procedure, other diagnostic eye procedures, class 2 ).

With APGs, most visits were grouped into either one of two medical APGs or one procedure APG. The most frequent APGs were APG 754 (eye disease except cataract, refraction disorder, and conjunctivitis) and APG 751 (cataracts), which together described in excess of two thirds of all visits.
Mean consultation times varied from 8.7 minutes for APG 948 (counselling) to 19.6 minutes for APG 294 (simple anterior segment eye procedures except for glaucoma). Average visit cost varied from $£ 3.90$ for APG 948 to $£ 30.33$ for APG 287 (minor ophthalmologic tests and procedures).

APGs grouped all visits at which a procedure took place into procedure groups. By contrast, the structure of the AVG grouper resulted in such visits being assigned to AVG procedure groups in only $25 \%$ of the cases: the remainder were assigned to diagnostic groups. Procedures that were always assigned to non-procedure AVG groups included visual field examination, dilate tear duct opening, revise eyelashes, eye examination and treatment, and rbc sedimentation rate. Other procedures, such as removal of foreign body, excision of chalazion, and repair of ectropion, and investigations such as electroretinograpy and anterior segment photography, were also assigned on the basis of diagnosis if the diagnosis was inappropriate, according to the AVG system, for the procedure or investigation.

The distribution of consultation times and costs for AVGs and APGs varied from group to group. Figure 1 shows examples of the distributions for cost and time according to AVGs and APGs. In general, the distributions were skewed to the left, and times were clustered around five, 10, and 15 minutes. To take account of the skew, the data were transformed by taking their logarithm before carrying out analysis of variance (ANOVA).

Table 3 shows the results from one way ANOVA of the dependent variables consulta-

Table 2 Most frequent ambulatory patient groups (APGs)

\begin{tabular}{|c|c|c|c|c|c|c|}
\hline \multirow[b]{2}{*}{$A P G$} & \multirow[b]{2}{*}{ Label } & \multirow[b]{2}{*}{$\%$ Visits } & \multicolumn{2}{|c|}{ Consultation time } & \multicolumn{2}{|l|}{ Cost } \\
\hline & & & Mean & $S D$ & Mean & $S D$ \\
\hline $754^{\star}$ & $\begin{array}{l}\text { Eye disease except cataract, refraction disorder and } \\
\text { conjunctivitis }\end{array}$ & 46.6 & 9.7 & 5.0 & 3.9 & 2.0 \\
\hline $751^{\star}$ & Cataracts & 20.7 & 11.5 & 6.2 & 4.7 & 2.4 \\
\hline 469 & Professional service & 13.3 & 10.6 & 5.5 & 7.6 & 5.1 \\
\hline $948^{\star}$ & Counselling & 2.5 & 8.7 & 5.5 & 3.9 & 2.1 \\
\hline 436 & Simple haematology tests & 1.9 & 13.5 & 7.8 & 9.0 & 3.2 \\
\hline 294 & $\begin{array}{l}\text { Simple anterior segment eye procedures except for } \\
\text { glaucoma }\end{array}$ & 1.5 & 19.6 & 4.2 & 9.8 & 1.4 \\
\hline 300 & Simple repair and plastic procedures of eye & 1.5 & 9.4 & 3.5 & 10.1 & 10.4 \\
\hline Other APGs & & 12.0 & 11.1 & 8.7 & 8.4 & 11.6 \\
\hline All visits & & 100.0 & 10.6 & 6.2 & 5.3 & 5.5 \\
\hline
\end{tabular}

* Percentage adjusted, includes only visits where no procedures were undertaken. 
Table 3 One way analysis of variance in resource use by AVGs, APGs, and individual clinician

\begin{tabular}{lllcccc}
\hline $\begin{array}{l}\text { Resource use } \\
\text { variable }\end{array}$ & $\begin{array}{l}\text { Independent } \\
\text { variable }\end{array}$ & $\begin{array}{l}\text { Mean sum of } \\
\text { squares }\end{array}$ & $\begin{array}{l}\text { Degrees of } \\
\text { freedom }\end{array}$ & F ratio & Significance & Adjusted $r^{2}$ \\
\hline Log time & AVG & 1.14 & 14307 & 3.78 & 0.000 & 0.108 \\
& APG & 0.66 & 7314 & 2.00 & 0.055 & 0.021 \\
Log cost & Clinician & 3.07 & 8313 & 11.43 & 0.000 & 0.206 \\
& AVG & 1.65 & 14307 & 4.53 & 0.000 & 0.095 \\
& APG & 3.38 & 7314 & 9.53 & 0.000 & 0.157 \\
& Clinician & 2.58 & 8313 & 7.05 & 0.000 & 0.131 \\
\hline
\end{tabular}

tion time and visit cost, using as explanatory variables the two case mix measures. AVGs explained a significant amount of variation in both variables, while APGs explained variation only in cost.

Table 3 also shows a one way ANOVA for time and cost using the individual clinician as the explanatory variable. This also explained a significant amount of the variance of both dependent variables. Indeed, for the time variable it explained more of the variance than either of the case mix measures. A two way ANOVA model was therefore fitted. The results were similar for both orders of model fitting; both case mix and clinician variables retained their ability to explain a significant amount of the variance. Indeed, the mean sums of squares (MSS) and the $F$ ratios actually increased for the time variable and for cost analysed by APG; for cost analysed by AVG the

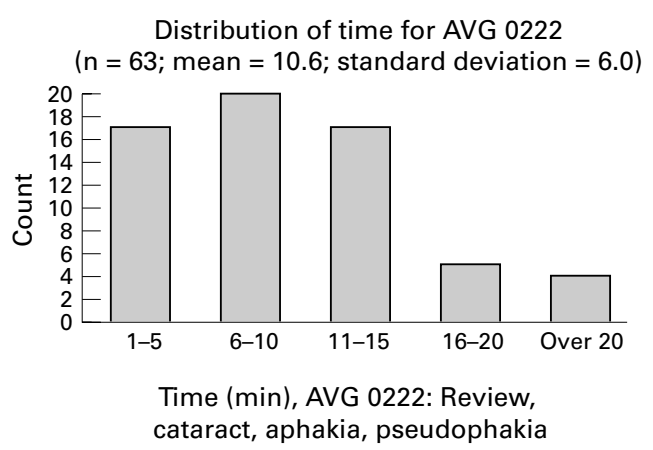

Distribution of time for APG 754 ( $n=196 ;$ mean $=10.0 ;$ standard deviation $=5.2$ )

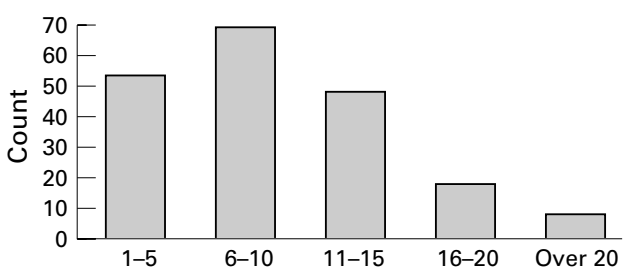

Time (min), APG 754: Eye disease except cataract, refraction disorder and conjunctivitis
MSS fell very slightly, although the $F$ ratios were higher. It may be concluded that the differences between clinicians could not be explained by differences in case mix.

Table 4 shows the two way model in which variation between clinicians was incorporated first into the model. The results, after controlling for differences between clinicians, are similar to those obtained in the one way ANOVA; AVGs performed better than APGs in explaining time variation, while the reverse was true for visit cost.

The influence of doctor grade and patient age on the length of consultation time was also investigated as a source of potential variation. Table 5 shows the wide variation between individual clinicians for a particular AVG. However, one way ANOVA showed that grade explained only $7.5 \%$ of the variation in time, and there was no significant correlation be-

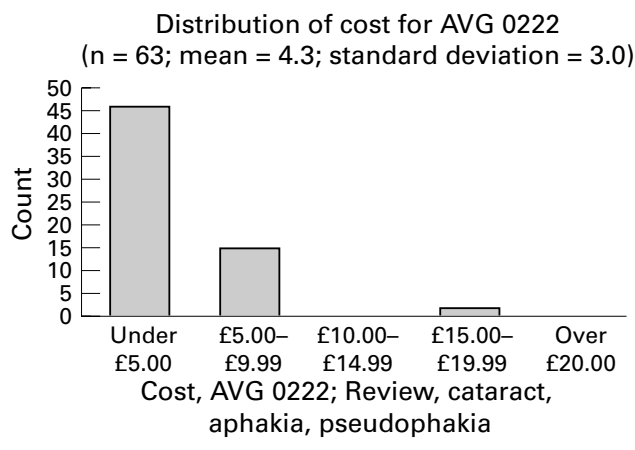

Distribution of cost for APG 754 ( $n=196 ;$ mean $=5.1 ;$ standard deviation $=5.1$ )

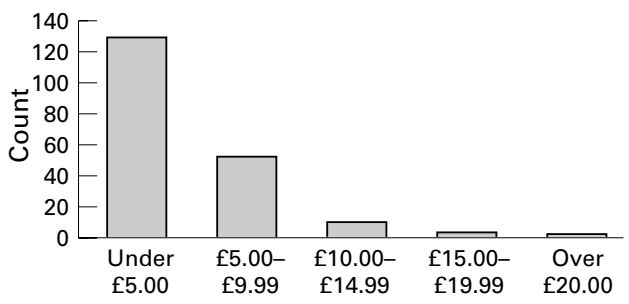

Cost, APG 754: Eye disease except cataract, refraction disorder and conjunctivitis

Figure 1 Frequency distributions of time and cost for Ambulatory Visit Group 0222 and Ambulatory Patient Group 754.

Table 4 Analysis of variance in resource use by AVGs and APGs adjusting for differences between clinicians

\begin{tabular}{|c|c|c|c|c|c|c|}
\hline $\begin{array}{l}\text { Resource use } \\
\text { variable }\end{array}$ & $\begin{array}{l}\text { Independent } \\
\text { variable }\end{array}$ & $\begin{array}{l}\text { Mean sum of } \\
\text { squares }\end{array}$ & $\begin{array}{l}\text { Degrees of } \\
\text { freedom }\end{array}$ & F ratio & Significance & Adjusted $r^{2}$ \\
\hline \multirow[t]{4}{*}{ Log time } & Clinician & 3.21 & 8299 & 14.31 & 0.000 & 0.338 \\
\hline & AVG & 1.22 & 14299 & 5.44 & 0.000 & \\
\hline & Clinician & 3.09 & 8306 & 11.91 & 0.000 & 0.234 \\
\hline & APG & 0.68 & 7306 & 2.63 & 0.012 & \\
\hline \multirow[t]{4}{*}{$\log \cos t$} & Clinician & 2.26 & 8299 & 7.18 & 0.000 & 0.253 \\
\hline & AVG & 1.47 & 14299 & 4.67 & 0.000 & \\
\hline & Clinician & 3.01 & 8306 & 10.21 & 0.000 & 0.301 \\
\hline & APG & 3.49 & 7306 & 11.86 & 0.000 & \\
\hline
\end{tabular}


Table 5 Average consultation times and average age of patients seen by clinicians within AVG 0223 (return patients, other anterior segment and optic nerve)

\begin{tabular}{lccl}
\hline Clinician & Observations & Mean consultation time & Mean patient age \\
\hline Consultant 1 & 9 & 10.3 & 65.6 \\
Consultant 2 & 30 & 6.6 & 67.0 \\
Consultant 3 & 14 & 8.4 & 72.4 \\
Clinical asst 1 & 12 & 15.3 & 63.9 \\
Clinical asst 2 & 8 & 13.2 & 70.7 \\
Clinical asst 3 & 11 & 9.0 & 74.8 \\
SHO 1 & 7 & 7.7 & 54.3 \\
SHO 2 & 5 & 14.6 & 73.6 \\
\hline
\end{tabular}

Three missing observations are excluded where details of clinician were unrecorded.

tween age and consultation time $(r=0.06$; $\mathrm{p}=0.24$ ).

Because cost data are difficult to collect, we explored the relation between consultation time and total cost. Clinician time costs accounted for $65 \%$ of total cost and there was a significant correlation between time and cost $(r=0.46 ; \mathrm{p}=0.00)$. Nurse time and costs were collected for 141 hospital outpatient visits. There was no statistically significant relation between nurse time and consultation time $(r=$ $0.03)$ nor visit cost $(r=0.08)$.

The visit type categories that we constructed displayed little variation in resource use. Most categories consumed similar resources, except for new attendances and follow up after procedure visits, which had slightly higher consultation times than other types of visit, and attendances for follow up after diagnostic assessment, which were slightly more costly than other types of visit. ANOVA showed that these categories could explain only $5.6 \%$ of the variation in consultation time and $7.6 \%$ of the cost variation.

\section{Discussion}

This study aimed to test to what extent AVGs and APGs could explain differences in outpatient consultation times and visit costs. AVGs were found to be capable of explaining a statistically significant amount of variation in both consultation times and costs, while APGs could not explain variations in times, but were better than AVGs at explaining cost variation.

This finding is explicable by the nature and origins of the two systems. AVGs were constructed by primarily using clinician time as the measure of resource consumption, although other resources, such as selected ancillaries, were also taken into account. In contrast, APGs were developed for prospective reimbursement and used measures of total cost. The implication is that different measures are useful for different purposes. For AVGs and APGs, the $r^{2}$ figures compare well with studies conducted in the United States, where AVGs were found to explain approximately one third of cost variation. ${ }^{15} 16$

The relation between consultation time and total visit cost was high; clinician time costs accounted for almost two thirds of total costs, suggesting that consultation time accounts for the bulk of resource use for most outpatient visits. This finding is different from that of studies carried out in other specialties, ${ }^{14}$ but it does confirm the expectations of Eye Infirmary staff who, before data collection, felt that nonclinical staff costs would be less important. For this specialty therefore, it is reasonable to assume that the statistical performance of both case mix measures can be judged by ability to explain differences in clinical staff time or visit costs.

On this basis however, the results seem to give mixed messages concerning the use of case mix. The total cost variance explained by APGs compares well with other studies and is higher than AVGs, which might suggest that APGs would be the system of choice for contracting and payment. Such a conclusion would be premature, however. First of all, the poor performance of APGs with respect to time suggests that, in ophthalmology, AVGs would be a better alternative for purposes other than contracting, such as clinical audit. Secondly, statistical performance is not the only criterion for choosing a system. An important point concerns the effort required to use APGs on a routine basis. Because cases can be assigned multiple procedure APGs, the system requires the user to specify decision rules to generate payment incentives, for example to ensure that unnecessary procedures are not encouraged or necessary procedures discouraged. This adds a considerable degree of complexity to the system.

The evaluation of patient visit type categories as potential contract categories indicated that there was too little variation in resource use between the different groups to warrant their use. This is important because such simple and convenient case categories would be an attractive prospect for outpatient contracting. It may be that it is possible to devise an approach that is halfway between this and a full case mix measure, as represented by AVGs and APGs, entailing aggregation of procedures into particular cost bands. For example, using the information from tables 1 and 2, procedures could be aggregated into two or three different groups, with four expensive/new or review, inexpensive/new or review bands for nonprocedure cases. An advantage of this is that it would not require collection of diagnostic data. However, this needs further testing of the type that we have carried out.

An important set of findings is that the impact of clinical variation on consultation time and cost differences is significant; that the way that different clinicians practice is as important in influencing the cost of an outpatient visit as differences between patients; that individual clinicians' consultation times and visit cost consistently differ when confronted with cases of a similar type, as defined by AVG and APG; and that other factors that might influence clinician variability, such as patient age and clinician grade, in fact have little effect. This further confirms the finding by Milne et al for rheumatology, ${ }^{14}$ that variation in clinical practice is a very important influence on the management of NHS outpatient resources.

This demonstration of the importance of clinical variability does not mean, however, that the value of case mix measurement is reduced. On the contrary, our results show the power of case mix measures to show that such variability 
KEY POINTS

- Changes in delivery of health care have resulted in greater use of outpatient services

- Case mix measures such as Ambulatory Visit Groups (AVGs) and Ambulatory Patient Groups (APGs) provide a means of classifying outpatient activity.

- Both measures explained significant levels of cost variation in ophthalmology outpatient visits.

- There were significant differences in consultation times and costs between clinicians that was not explained by differences in case mix.

- Existing measures may be useful for contracting and clinical audit, although data requirements are likely to preclude their routine use.

is due to differences not in case mix, but in factors such as clinicians' style and efficiency. This gives a valuable starting point for the evaluation of clinical practice, although of course it is partial in itself, excluding, for example, the issue of outcomes.

Both AVGs and APGs seem to perform reasonably well, bearing in mind that neither was designed with UK conditions in mind. They do provide useful information at a patient based level on outpatient workload and cost, which simpler measures fail to do. The main problems are the costs of data collection and administration, which are probably large enough to preclude the implementation of such measures in the near future. However, given the continuing shift of services from the acute sector to provision in day case and outpatient settings, the size of data collection costs relative to resource expenditure in ambulatory care may diminish. Therefore, growing interest in such systems and the value of understanding case mix in ambulatory care is almost certain to continue.

We would like to thank the National Casemix Office of the NHS Executive for financial support, Ms Dena Portman and other finance staff at Sunderland District General Hospital, Mrs Sharon Currie of Sunderland Eye Infirmary and all other clinicians and staff at the hospital who participated in the project. Any errors and all expressions of opinion are, however, the responsierrors and all expressions of
bility solely of the authors.

Funding: National Casemix Office, NHS Executive. Conflicts of interest: none.

1 Bardsley M, Coles J, Jenkins L. DRGs and health care. The management of case mix. London: King's Fund, 1989.

2 National Casemix Office. Healthcare Resource Groups (Version 1.0). Winchester: NHS Management Executive, 1991.

3 Perrin J. The capture of ambulatory care data: a review of the American system. Report commissioned by the Resource Management Unit of the Department of Health and North
West Thames Regional Health Authority, 1991.

4 McNamee P, Hutchinson A, Parkin D, Milne E, Steen N. Outpatient case mix: a survey of user requirements. Fournal of Management in Medicine 1994;8:29-37.

5 Schneider K, Lichtenstein J, Fetter R, Freeman J, Newbold R. The new ICD-9-CM Ambulatory Visit Groups classification scheme. New Haven: Yale University, 1986.

6 Averill R, Goldfield N, McGuire T, et al. Ambulatory Patient Groups definitions manual. Wallingford: $3 \mathrm{M}$ Health Information Systems, 1991.

7 Hutchinson A, Parkin D, Philips P. Case mix measures for ambulatory care. F Public Health Med 1991;13:189-97.

8 Lion J, Vertrees J, Malbon A, Harrow B, Collard A, Mowschenson $\mathrm{P}$. The case mix of ambulatory surgery as measured by Ambulatory Visit Groups. Fournal of Ambulatory Care Management 1990;13:33-45.

9 Sulvetta M. Achieving cost control in the hospital outpatient department. Health Care Financing Review 1991; Annual Supplement: $95-106$.

10 Lagaida R, Hindle D. A casemix classification for hospitalbased ambulatory services. Report commissioned by the New
South Wales Health Department, Sydney, Australia, 1992.

11 Sanderson H, Reeves C, Ross P. Counting the cost. British fournal of Health Care Computing and Information Management 1993;10:13-14.

12 Anthony P. Healthcare Resource Groups in the NHS: a measure of success. Public Finance and Accountancy 1993;23 April:8-10

13 American Medical Association. CPT 1991: Physicians' Current Procedural Terminology. Chicago: AMA, 1991.

14 Milne E, Hutchinson A, Platt P, Parkin D. An evaluation of Ambulatory Visit Groups in rheumatology outpatients. $\mathcal{F}$ Public Health Med 1994;16:41-9.

15 Lion J, Henderson M, Bergman A, Malbon A. Ambulatory Visit Groups: how they perform for oncology outpatient departments. Fournal of Cancer Program Management 1987; 2:22-33.

16 Hornbrook M, Johnson R, Hurtado A. Validation of Ambulatory Visit Groups on an HMO database. Development of an
ambulatory patient classification system. New Haven: Yale University, 1987. 